\title{
Improved production of poly(lactic acid)-like polyester based on metabolite analysis to address the rate-limiting step
}

\author{
Ken'ichiro Matsumoto ${ }^{1,2^{*}}$, Kota Tobitani ${ }^{1}$, Shunsuke Aoki ${ }^{1}$, Yuyang Song ${ }^{1,3}$, Toshihiko Ooi ${ }^{1,4}$ and Seiichi Taguchi ${ }^{1,4^{*}}$
}

\begin{abstract}
The biosynthesis of poly(lactic acid) (PLA)-like polymers, composed of $>99$ mol\% lactate and a trace amount of 3-hydroxybutyrate, in engineered Corynebacterium glutamicum consists of two steps; the generation of the monomer substrate lactyl-coenzyme A (COA) and the polyhydroxyalkanoate (PHA) synthase-catalyzed polymerization of lactyl-CoA. In order to increase polymer productivity, we explored the rate-limiting step in PLA-like polymer synthesis based on quantitative metabolite analysis using liquid chromatography mass spectroscopy (LC-MS). A significant pool of lactyl-CoA was found during polymer synthesis. This result suggested that the rate-limitation occurred at the polymerization step. Accordingly, the expression level of PHA synthase was increased by means of codon-optimization of the corresponding gene that consequently led to an increase in polymer content by 4.4-fold compared to the control. Notably, the codon-optimization did not significantly affect the concentration of lactyl-CoA, suggesting that the polymerization reaction was still the rate-limiting step upon the overexpression of PHA synthase. Another important finding was that the generation of lactyl-CoA was concomitant with a decrease in the acetyl-CoA level, indicating that acetyl-CoA served as a CoA donor for lactyl-CoA synthesis. These results show that obtaining information on the metabolite concentrations is highly useful for improving PLA-like polymer production. This strategy should be applicable to a wide range of PHA-producing systems.
\end{abstract}

Keywords: Biobased plastic; P(LA-co-3HB); Polyhydroxybutyrate; Metabolome analysis

\section{Introduction}

Bacterial polyesters polyhydroxyalkanoates (PHAs) are synthesized via the supply of monomer hydroxyacyl-CoA molecules and polymerization of the monomers catalyzed by PHA synthases (Rehm 2003; Matsumoto and Taguchi 2013b; Lu et al. 2009). The rate-limiting step in PHA synthesis may be either the monomer supply or polymerization, which can vary depending on the combination of the relevant enzymes, production hosts and carbon source. To date, the rate-limiting step has been estimated by modulating the activity of each step to see its effect on polymer productivity (Jung et al. 2000; Kichise et al. 1999; Taguchi et al. 2001). This indirect approach, however, was unable to provide quantitative information on the

\footnotetext{
* Correspondence: mken@eng.hokudai.ac.jp; staguchi@eng.hokudai.ac.jp 'Division of Biotechnology and Macromolecular Chemistry, Graduate School of Engineering, Hokkaido University, N13-W8, Kita-ku, Sapporo 060-8628, Japan

${ }^{4}$ CREST, JST, 4-1-8 Honcho, Kawaguchi, Saitama 332-0012, Japan Full list of author information is available at the end of the article
}

metabolic pathways. The aim of this study was to determine the intracellular concentration of the metabolic intermediates in the PHA biosynthetic pathways in order to explore the rate-limiting step.

In this study, we chose the PLA-like polymer-producing Corynebacterium glutamicum as the target (Song et al. 2012). This microorganism, which is known as an industrial amino acid producer with GRAS (Generally Regarded As Safe) status, has been engineered to express three exogenous genes encoding D-lactate dehydrogenase, propionyl-CoA transferase (PCT) and LA-polymerizing PHA synthase ( $\mathrm{PhaCl}_{\mathrm{PS}} \mathrm{STQK}$ ) (Taguchi and Doi 2004; Taguchi et al. 2008) (see pathway in Figure 1). This unique bacterial system was shown to be capable of producing PLA-like polymer directly from glucose via one-pot fermentation. It should be noted that the PLA-like polymer consists of $>99 \mathrm{~mol} \% \mathrm{LA}$ and a trace amount of 3hydroxybutyrate (3HB) (Song et al. 2012), and thus is here referred to as PLA'. The challenge of the system was that 


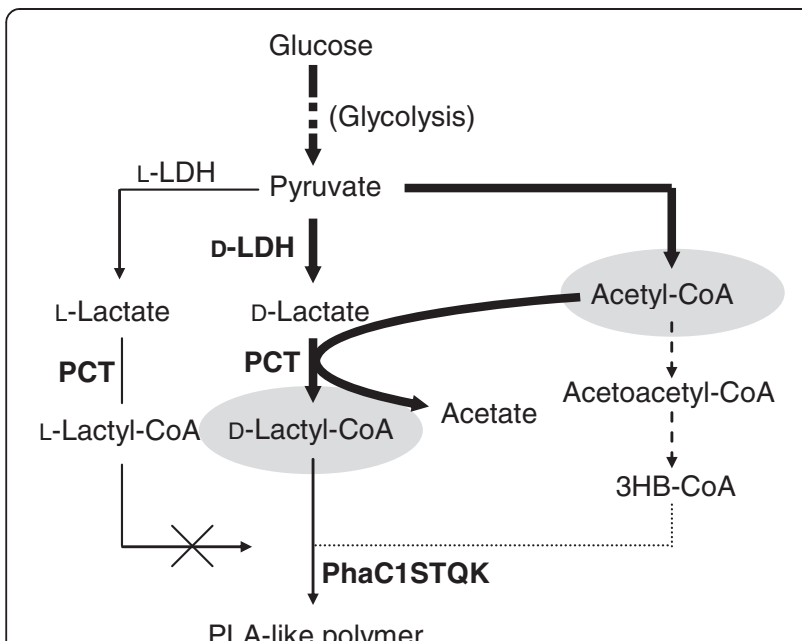

PLA-like polymer

Figure 1 Metabolic pathway for PLA-like polymer production in engineered C. glutamicum. PLA-like polymers contained $<1 \mathrm{~mol} \%$ 3 HB units. L-LDH, L-lactate dehydrogenase; D-LDH, D-lactate dehydrogenase; PCT, propionyl-CoA transferase; PhaC1STQK, lactate-polymerizing PHA synthase. The bold letters indicate exogenous enzymes. The dashed lines indicate the putative pathways. The dotted line indicates a very weak pathway. The gray ovals indicate significantly pooled metabolites.

had to be overcome was the low productivity of the polymer $(0.03 \mathrm{~g} / \mathrm{L})$ compared to typical bacterial PHAs.

To meet this challenge, we attempted to identify the rate-limiting step in PLA' synthesis by means of quantitative metabolite analysis using liquid chromatography mass spectroscopy (LC-MS) (Zhou et al. 2012). This method was reported to be suitable for measuring the derivatives of CoA having a relatively high molecular weight and polarity, while gas chromatography-MS has been used for detecting relatively low-polarity and small molecules (Poblete-Castro et al. 2012). In this study, the concentrations of the important intermediates for PLA' production were determined, i.e. lactyl-CoA, acetyl-CoA, acetoacetyl-CoA and 3HB-CoA (Figure 1). To the best of our knowledge this was the first successful monitoring of the intracellular CoA-derivatives during PLA' production, which was realized by a rational metabolic engineering approach.

\section{Materials and methods \\ Plasmid construction}

The four oligonucleotides 5 '-gtcaccggatcccggttaactctag-3', $5^{\prime}$-actcgagcctgcaggagatcttcgatatca-3', 5' -ctagtgatatcgaagat ctcctgc- $3^{\prime}$ and $5^{\prime}$-aggctcgagtctagagttaaccgggatccg- $3^{\prime}$ were inserted into the XbaI/BstEII sites of pPSPTG1 (Song et al. 2012) so as to create BamHI, HpaI, XbaI, XhoI, Sbfl, $B g l \mathrm{II}$ and EcoRV sites (pPSDCP). Fragments of the $l d h A$ gene from Escherichia coli (gene ID: 12930508), the pct gene from Megasphaera elsdenii and the phaC1STQK gene (Taguchi et al. 2008) were inserted into pPSDCP at

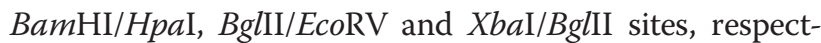
ively, to yield pPSldhAC1STQKpct. The codon-optimized phaC1STQK gene [ephaC1STQK, accession No. AB983346 (DDBJ)] was chemically synthesized (Eurofins Genomics) for the expression in C. glutamicum and its XbaI/Sbfl fragment was inserted in a similar manner so as to yield pPSldhAeC1STQKpct (Additional file 1: Table S1).

\section{Strain, culture conditions and polymer analysis}

C. glutamicum ATCC13803 was transformed by electroporation, as described previously (Liebl et al. 1989). For polymer production, the engineered strains were grown in $2 \mathrm{ml}$ nutrient-rich CM2G medium (Kikuchi et al. 2003) at $30^{\circ} \mathrm{C}$ for $24 \mathrm{~h}$ with reciprocal shaking at 180 strokes/ $\mathrm{min}$. Two hundred microliters of the preculture were then transferred into $2 \mathrm{~mL}$ minimal MMTG medium (Kikuchi et al. 2003) containing $60 \mathrm{~g} / \mathrm{L}$ glucose and $0.45 \mathrm{mg} / \mathrm{L}$ of biotin, and further cultivated for $72 \mathrm{~h}$ at $30^{\circ} \mathrm{C}$. When needed, kanamycin $(50 \mu \mathrm{g} / \mathrm{mL})$ was added to the medium. After cultivation, cells were lyophilized for polymer extraction. The polymer content was determined using gas chromatography as described previously (Takase et al. 2004). Based on this analytical method, 3 HB units in the polymer were below the detection limit.

\section{LC-MS analysis}

The cell extract was prepared using a method modified from a previous report (Kiefer et al. 2011). The cells were cultivated on $2 \mathrm{~mL}$ MMTG medium as described above, then harvested at $18 \mathrm{~h}$. The cells were resuspended in $200 \mu \mathrm{L}$ of chilled water, then combined with $1 \mathrm{~mL}$ chilled acetonitrile containing $0.1 \mathrm{M}$ formic acid and treated with sonication for $5 \mathrm{sec} \times 5$ times. The supernatant was transferred to a new microtube and evaporated in vacuo at $4{ }^{\circ} \mathrm{C}$. The sample was dissolved in $200 \mu \mathrm{L}$ of chilled water. LC-MS analysis was performed using an LCMS-8030 (Shimadzu) equipped with a Mastro C18 column $(150 \mathrm{~mm})$, electrospray ionization (ESI) and triple quadrupole mass spectroscopy. Carrier A: $5 \mathrm{mM}$ ammonium acetate $(\mathrm{pH}$ 5.6) containing $5 \mathrm{mM}$ dimethylbutylamine (Gao et al. 2007) and carrier B: methanol were used with a flow rate of $0.2 \mathrm{~mL} / \mathrm{min}$ in gradient mode, as follows: 0 min, 10\% B; 3 min, 10\% B; 15 min, 95\% B; $18 \mathrm{~min}$, $95 \% \mathrm{~B} ; 23 \mathrm{~min}, 10 \% \mathrm{~B}$. The ESI voltage was $3.5 \mathrm{kV}$ in the negative mode. Nitrogen was used as a nebulizer $(3.0 \mathrm{~mL} /$ $\mathrm{min})$ and drying gas $(15.0 \mathrm{~mL} / \mathrm{min}) .[\mathrm{M}-\mathrm{H}]^{-}$ions from acetyl-CoA $(\mathrm{m} / \mathrm{z}=808$, retention time: $9.1 \mathrm{~min})$, acetoacetyl-CoA $(\mathrm{m} / \mathrm{z}=850, \mathrm{rt}: 8.9 \mathrm{~min}), 3 \mathrm{HB}-\mathrm{CoA}(\mathrm{m} / \mathrm{z}=$ 852, rt: $9.1 \mathrm{~min})$ and lactyl-CoA $(\mathrm{m} / \mathrm{z}=838, \mathrm{rt}: 8.7 \mathrm{~min})$ were monitored using the selected ion monitoring mode. Acetyl-CoA, acetoacetyl-CoA and 3HB-CoA, used as standards, were purchased from Sigma Aldrich. Lactyl-CoA was synthesized via CoA-transferring reaction by $\mathrm{PCT}$, as follows. The reaction mixture containing $100 \mathrm{mM}$ Tris- 
$\mathrm{HCl}$ (pH 7.4), $0.4 \mathrm{mM}$ acetyl-CoA, $12.5 \mathrm{mM}$ sodium lactate and $0.1 \mathrm{mg} / \mathrm{mL}$ purified His-tagged PCT (Additional file 1) was incubated at $30^{\circ} \mathrm{C}$ for $30 \mathrm{~min}$. Then lactyl-CoA was purified using a preparative HPLC equipped with a C18 reverse phase column.

\section{SDS-PAGE analysis}

The cells cultivated under the polymer producing conditions were harvested at $18 \mathrm{~h}$. The cells were resuspended in $25 \mathrm{mM}$ Tris $-\mathrm{HCl}(\mathrm{pH} \mathrm{7.5)}$ buffer and treated with sonication. The whole cell extracts were subjected to SDS-PAGE.

\section{Results}

The use of a single plasmid system increased transformation efficiency

In a previous study, a dual plasmid system using pPS and $\mathrm{pVC}$ vectors was used for PLA' production (Song et al. 2012). To reduce the use of antibiotics, the expression vector was reconstructed so that the recombinant form was maintained only in the presence of kanamycin. In addition, the single plasmid system improved the transformation efficiency compared to the dual plasmid system (from $3 \times 10^{2}$ to $2 \times 10^{4}$ colonies/ $\mu \mathrm{g} \mathrm{DNA}$ ). Therefore, the single plasmid system was used for further study.

\section{Determination of the CoA intermediates in the engineered C. glutamicum}

The wild-type C. glutamicum and the recombinant cells harboring pPSldhAC1STQKpct were cultivated under the polymer-producing conditions. The cells were harvested in the logarithmic growth phase (18 h) and subjected to metabolite analysis. The concentrations of aectyl-CoA, lactyl-CoA, acetoacetyl-CoA and 3HB-CoA in the cells were determined. The key points for the successful measurement of the CoA derivatives were shown to be a rapid extraction of the cells along with the an appropriate ion pair reagent. For the wild-type cells, the acetyl-CoA concentration was $89 \mathrm{nmol} / \mathrm{g}$-dry cells, and unexpectedly, a small amount of lactyl-CoA was also observed (Figure 2). In contrast, the recombinant cells exhibited an elevated concentration of lactyl-CoA, which was concomitant with a significant reduction in the concentration of acetyl-CoA. This result suggests that PCT promoted lactyl-CoA synthesis, and more importantly, acetylCoA is able to serve as a CoA donor for the generation of lactyl-CoA (Figure 1). The concentrations of acetoacetyl$\mathrm{CoA}$ and $3 \mathrm{HB}-\mathrm{CoA}$ were below the detection limit for all of the conditions tested (data not shown). From these observations, the interconversion of lactate + acetyl-CoA $\leftrightarrow$ lactyl-CoA + acetate presumably achieved an equilibrium state, namely, the polymerization of lactyl-CoA would be a retarded step.

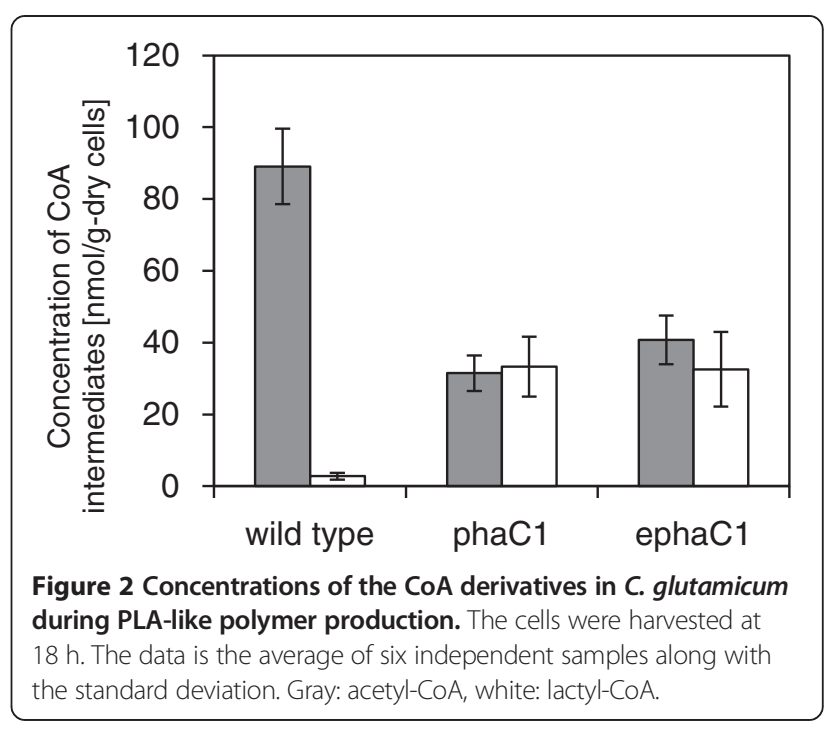

Enhanced expression of PHA synthase elevated PLA-like polymer production

In order to evaluate the aforementioned hypothesis and to overcome the existing limitation, we attempted to improve the expression level of PHA synthase. For this purpose, the codon-optimized PHA synthase gene ephaC1 $1_{\mathrm{Ps}} \mathrm{STQK}$, which was supposed to be efficiently translated in $C$. glutamicum, was synthesized. First, the effect of the codon-optimization on the expression of the enzyme was evaluated. As shown in Figure 3, the cells harboring the codon-optimized gene had an increase in the expression level of PHA synthase.

Next, the PLA' production was investigated for the cells harboring parent and codon-optimized PHA synthase genes. As expected, the cells expressing a higher level of PHA synthase accumulated more PLA' (4.4-fold) than the control (Table 1). Thus, the metabolic engineering, which was designed based on the metabolite analysis, did successfully improve the polymer production. In addition, this result supported the validity of the method for the determination of the metabolite concentrations.

\section{Metabolite analysis of the modified cells indicated the rate-limiting step remained}

The metabolite concentrations in the cells harboring the codon-optimized gene were measured. Despite the increase in the polymer content (Table 1), there was no significant difference between the metabolite levels in the two types of recombinant cells (Figure 2). This result indicated that the rate-limiting at polymerization step remained even with the reinforced expression of PHA synthase.

\section{Discussion}

In this study, metabolite analysis was shown to provide quantitative information that was very useful for addressing the rate-limiting step in the PLA' production. In the 


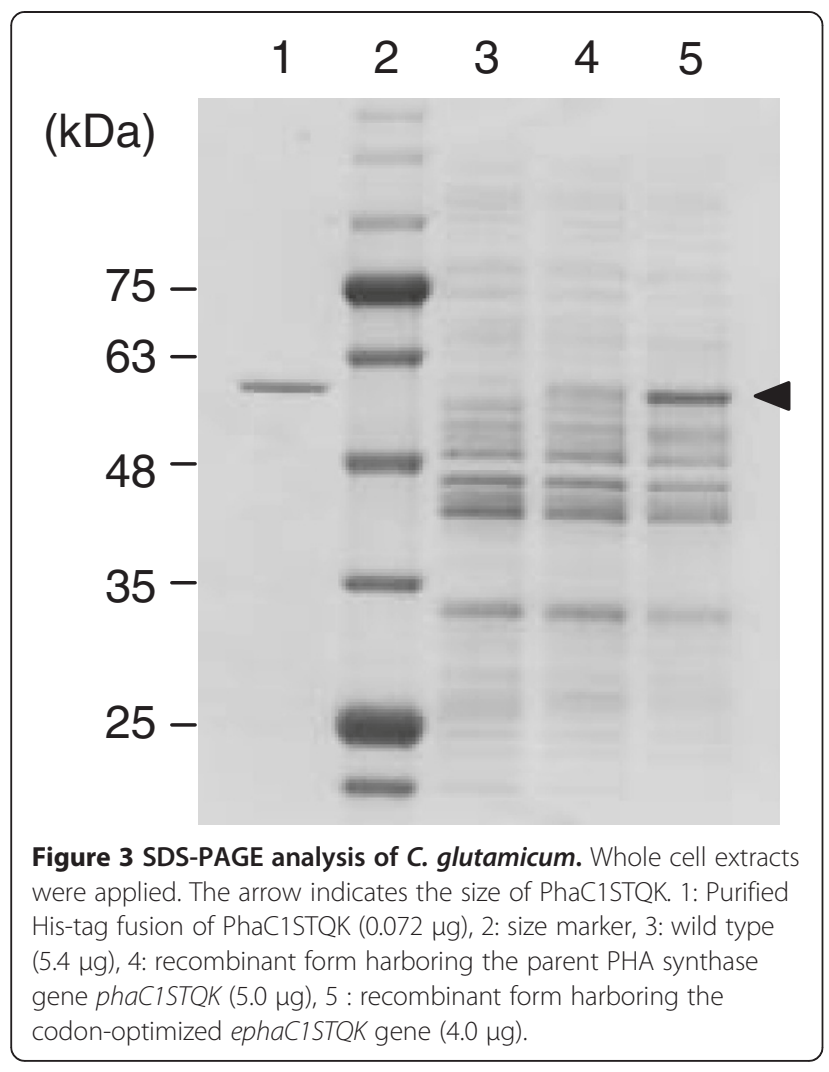

previous studies, the rate-limiting step in PHA production has been only qualitatively explored based on the polymer production in vivo. For examples, in the case of $\mathrm{P}(3 \mathrm{HB})$ production in C. glutamicum, the expression of the highly active acetoacetyl-CoA reductase (PhaB) mutant from Ralstonia eutropha improved $\mathrm{P}(3 \mathrm{HB})$ production (Matsumoto et al. 2013), suggesting that monomer supply is a rate-limiting step in $\mathrm{P}(3 \mathrm{HB})$ synthesis. A similar result was obtained in transgenic $\mathrm{P}(3 \mathrm{HB})$-producing tobacco (Matsumoto et al. 2011). Here it should be noted that in these studies it was impossible to determine whether the improved the PhaB activity was sufficient or not unless the $\mathrm{PhaB}$ activity was further increased. In other words, $\mathrm{P}$ $(3 \mathrm{HB})$ production should reach a plateau if the PhaB activity was sufficient under the original conditions. In contrast, based on the metabolite analysis, the rate-limitation of PLA

Table 1 PLA-like polymer production in engineered C. glutamicum ${ }^{\mathrm{a}}$

\begin{tabular}{lll}
\hline Relevant genes & $\begin{array}{l}\text { Cell dry weight } \\
\mathbf{( g / L )}\end{array}$ & $\begin{array}{l}\text { Polymer content } \\
\text { (wt\%) }\end{array}$ \\
\hline None (wild-type control) & $11.4 \pm 1.7$ & $\mathrm{ND}^{\mathrm{b}}$ \\
\hline phaC1STQK, pct, IdhA & $10.7 \pm 2.0$ & $0.27 \pm 0.01$ \\
\hline ephaC1STQK, pct, IdhA & $9.3 \pm 0.5$ & $1.19 \pm 0.01$ \\
\hline
\end{tabular}

${ }^{a}$ Cells were grown on $2 \mathrm{~mL}$ MMTG medium containing glucose for $72 \mathrm{~h}$ at $30^{\circ} \mathrm{C}$. The data is the average of triplicate samples along with the standard deviation. ${ }^{\mathrm{b}} \mathrm{ND}$ : not detected. synthesis at polymerization step was shown to remain after PHA synthase was overexpressed. Therefore, it was expected that an additional enhancement in polymerizing activity would be needed to increase PLA' production.

It was a chance discovery that the engineered $C$. glutamicum expressing PhaC $1_{\mathrm{Ps}} \mathrm{STQK}$ synthesized PLA'. On the other hand, it has been reportedly demonstrated that $E$. coli engineered to express the same set of enzymes did not produce PLA' (Nduko et al. 2013; Shozui et al. 2011; Yamada et al. 2009; Matsumoto and Taguchi 2013a). Thus, the mechanism for PLA' synthesis in C. glutamicum has been an important issue. A clue for answering this question might be the small amount of $3 \mathrm{HB}$ units $(<1 \mathrm{~mol}$ $\%$ ) incorporated into the polymer (Song et al. 2012). The presence of $3 \mathrm{HB}$ units in the polymer suggests that this organism is likely to possess intrinsic 3HB-CoA (Figure 1). The result of the present study, however, demonstrated that the concentration of $3 \mathrm{HB}-\mathrm{CoA}$ was below the detection limit. Thus, 3HB-CoA may be synthesized via an unidentified, very weak route and/or rapidly metabolized. In comparison, in R. eutropha, which is an efficient $\mathrm{P}(3 \mathrm{HB})$ producer, a much higher concentration of $3 \mathrm{HB}-\mathrm{CoA}$ (0.1-1 nmol/g-dry cells) has been reportedly observed (Fukui et al. 2014). The very low 3HB-CoA level in $C$. glutamicum might account for the capacity of this organism to produce the copolymer with an extremely high LA fraction. Further investigation of LA-based polymerproducing $E$. coli is needed to clarify this issue.

The wild-type C. glutamicum unexpectedly synthesized lactyl-CoA or another compound having the same $\mathrm{m} / \mathrm{z}$ and retention time. The metabolite level was too low to be detected using the MS/MS mode. Thus, currently the molecule is not confidently identified. However, if the existence of lactyl-CoA is postulated, this molecule should be L-lactyl-CoA, which cannot be incorporated into the polymer due to the strict stereospecificity of PHA synthase (Tajima et al. 2009), because the wild-type C. glutamicum possesses no D-LDH gene (Kalinowski et al. 2003). To date, the presence of lactyl-CoA in C. glutamicum has not been reported and its physiological role is unknown. Thus it may be an interesting research target.

Although PLA' production was successfully increased by the overexpression of PHA synthase (Table 1), the polymer content was lower than previously reported results (up to $1.4 \mathrm{wt} \%$ ) obtained using100 mL-scale flask cultures (Song et al. 2012). This difference was probably due to the aeration efficiencies in the test tubes and flasks. Because the lactic acid production in C. glutamicum is influenced by the oxygen supply (Inui et al. 2004), the aeration rate would be expected to have an impact on PLA' production. More detailed analysis and fine-tuning of the aeration using a jar-fermentor is needed to optimize the culture conditions.

In summary, the levels of CoA derivatives in engineered C. glutamicum during PLA' production were determined, 
which allowed us to identify a rate-limitation at the polymerization step. In fact, overexpression of PHA synthase successfully increased the polymer production. In addition, acetyl-CoA probably served as a CoA donor for supplying lactyl-CoA in C. glutamicum.

\section{Additional file}

Additional file 1: Protocol for purification of PCT.

\section{Competing interests}

The authors declare that they have no competing interests.

\section{Author contributions}

KM designed the study. KM wrote the manuscript TO and ST participated herein. KT performed the experiment work and developed LC-MS method. SA synthesized lactyl-CoA. YS developed protocols to manipulate C. glutamicum All authors read and approved the submission of the manuscript.

\section{Acknowledgements}

This work was supported by Japans Society for the Promotion of Science (JSPS) Kakenhi (No. 26281043 to K.M., Nos. 23310059 and 26660080 to S.T. and No. 23580452 to T.O.), Japan Science and Technology Agency (JST), Precursory Research for Embryonic Science and Technology (PRESTO) and JST, CREST. Pacific Edit reviewed the manuscript prior to submission.

\section{Author details}

Division of Biotechnology and Macromolecular Chemistry, Graduate School of Engineering, Hokkaido University, N13-W8, Kita-ku, Sapporo 060-8628, Japan. ${ }^{2}$ PRESTO-JST, K's Gobancho, Building 7, Gobancho Chiyoda-ku, Tokyo 102-0076, Japan. ${ }^{3}$ College of Enology, Northwest A\&F University, 22 Xinong Road, Yangling, 712100 Shaanxi, China. ${ }^{4}$ CREST, JST, 4-1-8 Honcho, Kawaguchi, Saitama 332-0012, Japan.

\section{Received: 3 September 2014 Accepted: 6 November 2014}

Published online: 18 November 2014

\section{References}

Fukui T, Chou K, Harada K, Orita I, Nakayama Y, Bamba T, Nakamura S, Fukusaki E (2014) Metabolite profiles of polyhydroxyalkanoate-producing Ralstonia eutropha H16. Metabolomics 10(2):190-202. doi:10.1007/s11306-013-0567-0

Gao L, Chiou W, Tang H, Cheng XH, Camp HS, Burns DJ (2007) Simultaneous quantification of malonyl-CoA and several other short-chain acyl-CoAs in animal tissues by ion-pairing reversed-phase HPLC/MS. J Chromatogr B 853(1-2):303-313. doi:10.1016/j.jchromb.2007.03.029

Inui M, Murakami S, Okino S, Kawaguchi H, Vertes AA, Yukawa H (2004) Metabolic analysis of Corynebacterium glutamicum during lactate and succinate productions under oxygen deprivation conditions. J Mol Microb Biotech 7(4):182-196. doi:10.1159/000079827

Jung Y, Park J, Lee Y (2000) Metabolic engineering of Alcaligenes eutrophus through the transformation of cloned phbCAB genes for the investigation of the regulatory mechanism of polyhydroxyalkanoate biosynthesis. Enzyme Microb Technol 26(2-4):201-208

Kalinowski J, Bathe B, Bartels D, Bischoff N, Bott M, Burkovski A, Dusch N, Eggeling L, Eikmanns BJ, Gaigalat L, Goesmann A, Hartmann M, Huthmacher K, Kramer R, Linke B, McHardy AC, Meyer F, Mockel B, Pfefferle W, Puhler A, Rey DA, Ruckert C, Rupp O, Sahm H, Wendisch VF, Wiegrabe I, Tauch A (2003) The complete Corynebacterium glutamicum ATCC 13032 genome sequence and its impact on the production of L-aspartate-derived amino acids and vitamins. J Biotechnol 104(1-3):5-25. doi:10.1016/50168-1656(03)00154-8

Kichise T, Fukui T, Yoshida Y, Doi Y (1999) Biosynthesis of polyhydroxyalkanoates (PHA) by recombinant Ralstonia eutropha and effects of PHA synthase activity on in vivo PHA biosynthesis. Int J Biol Macromol 25(1-3):69-77

Kiefer P, Delmotte N, Vorholt JA (2011) Nanoscale ion-pair reversed-phase HPLC-MS for sensitive metabolome analysis. Anal Chem 83(3):850-855. doi:10.1021/Ac102445r

Kikuchi Y, Date M, Yokoyama K, Umezawa Y, Matsui H (2003) Secretion of active-form Streptoverticillium mobaraense transglutaminase by Corynebacterium glutamicum: processing of the pro-transglutaminase by a cosecreted subtilisin-Like protease from Streptomyces albogriseolus. Appl Environ Microbiol 69(1):358-366

Liebl W, Bayerl A, Schein B, Stillner U, Schleifer KH (1989) High efficiency electroporation of intact Corynebacterium glutamicum cells. FEMS Microbiol Lett 53(3):299-303

Lu JN, Tappel RC, Nomura CT (2009) Mini-review: Biosynthesis of poly(hydroxyalkanoates). Polym Rev 49(3):226-248. doi:10.1080/15583720903048243

Matsumoto K, Taguchi S (2013a) Biosynthetic polyesters consisting of 2-hydroxyalkanoic acids: current challenges and unresolved questions. Appl Microbiol Biotechnol 97(18):8011-8021. doi:10.1007/s00253-013-5120-6

Matsumoto K, Taguchi S (2013b) Enzyme and metabolic engineering for the production of novel biopolymers: crossover of biological and chemical processes. Current Opinion in Biotechnology 24(6):1054-1060. doi:10.1016/j.copbio.2013.02.021

Matsumoto K, Morimoto K, Gohda A, Shimada H, Taguchi S (2011) Improved polyhydroxybutyrate (PHB) production in transgenic tobacco by enhancing translation efficiency of bacterial PHB biosynthetic genes. J Biosci Bioeng 111(4):485-488. doi:10.1016/j.jbiosc.2010.11.020

Matsumoto K, Tanaka Y, Watanabe T, Motohashi R, Ikeda K, Tobitani K, Yao M, Tanaka I, Taguchi S (2013) Directed evolution and structural analysis of NADPH-dependent acetoacetyl Coenzyme A (acetoacetyl-CoA) reductase from Ralstonia eutropha reveals two mutations responsible for enhanced kinetics. Appl Environ Microbiol 79(19):6134-6139. doi:10.1128/Aem. 01768-13

Nduko JM, Matsumoto K, Ooi T, Taguchi S (2013) Effectiveness of xylose utilization for high yield production of lactate-enriched P(lactate-co-3-hydroxybutyrate) using a lactate-overproducing strain of Escherichia coli and an evolved lactate-polymerizing enzyme. Metab Eng 15:159-166. doi:10.1016/j.ymben.2012.11.007

Poblete-Castro I, Escapa IF, Jaeger C, Puchalka J, Lam CMC, Schomburg D, Prieto MA, dos Santos VAPM (2012) The metabolic response of $P$. putida KT2442 producing high levels of polyhydroxyalkanoate under single- and multiple-nutrient-limited growth: Highlights from a multi-level omics approach. Microb Cell Fact 11:34. doi:10.1186/1475-2859-11-34

Rehm BHA (2003) Polyester synthases: natural catalysts for plastics. Biochem J 376:15-33

Shozui F, Matsumoto K, Motohashi R, Sun JA, Satoh T, Kakuchi T, Taguchi S (2011) Biosynthesis of a lactate (LA)-based polyester with a 96 mol\% LA fraction and its application to stereocomplex formation. Polym Degrad Stab 96(4):499-504. doi:10.1016/j.polymdegradstab.2011.01.007

Song YY, Matsumoto K, Yamada M, Gohda A, Brigham CJ, Sinskey AJ, Taguchi S (2012) Engineered Corynebacterium glutamicum as an endotoxin-free platform strain for lactate-based polyester production. Appl Microbio Biotechnol 93(5):1917-1925. doi:10.1007/s00253-011-3718-0

Taguchi S, Doi Y (2004) Evolution of polyhydroxyalkanoate (PHA) production system by "enzyme evolution": Successful case studies of directed evolution. Macromol Biosci 4(3):145-156

Taguchi S, Maehara A, Takase K, Nakahara M, Nakamura H, Doi Y (2001) Analysis of mutational effects of a polyhydroxybutyrate (PHB) polymerase on bacterial PHB accumulation using an in vivo assay system. FEMS Microbiol Lett 198(1):65-71

Taguchi S, Yamada M, Matsumoto K, Tajima K, Satoh Y, Munekata M, Ohno K, Kohda K, Shimamura T, Kambe H, Obata S (2008) A microbial factory for lactate-based polyesters using a lactate-polymerizing enzyme. Proc Natl Acad Sci USA 105(45):17323-17327

Tajima K, Satoh Y, Satoh T, Itoh R, Han XR, Taguchi S, Kakuchi T, Munekata M (2009) Chemo-enzymatic synthesis of poly(lactate-co-(3-hydroxybutyrate)) by a lactate-polymerizing enzyme. Macromolecules 42(6):1985-1989

Takase K, Matsumoto K, Taguchi S, Doi Y (2004) Alteration of substrate chain-length specificity of type II synthase for polyhydroxyalkanoate biosynthesis by in vitro evolution: in vivo and in vitro enzyme assays. Biomacromolecules 5(2):480-485

Yamada M, Matsumoto K, Nakai T, Taguchi S (2009) Microbial production of lactate-enriched poly[(R)-lactate-co-(R)-3-hydroxybutyrate] with novel thermal properties. Biomacromolecules 10(4):677-681

Zhou B, Xiao JF, Tuli L, Ressom HW (2012) LC-MS-based metabolomics. Mol Biosyst 8(2):470-481. doi:10.1039/C1mb05350g

\section{doi:10.1186/s13568-014-0083-2}

Cite this article as: Matsumoto et al.: Improved production of poly(lactic acid)-like polyester based on metabolite analysis to address the rate-limiting step. AMB Express 2014 4:83. 